\title{
Ventral Capsule/Ventral Striatum Deep Brain Stimulation Does Not Consistently Diminish Occipital Cross-Frequency Coupling
}

\author{
Alik S. Widge, Samuel Zorowitz, Katherine Link, Earl K. Miller, Thilo Deckersbach, Emad N. \\ Eskandar, and Darin D. Dougherty \\ Department of Psychiatry (ASW, SZ, TD, DDD), Massachusetts General Hospital and Harvard \\ Medical School, Charlestown; and Picower Institute for Learning \& Memory (ASW, EKM), \\ Massachusetts Institute of Technology, Cambridge, Massachusetts; The Johns Hopkins \\ University (KL), Baltimore, Maryland; and Department of Neurological Surgery (ENE), \\ Massachusetts General Hospital and Harvard Medical School, Boston, Massachusetts.
}

\section{To the Editor}

Bahramisharif et al. (1) reported that deep brain stimulation (DBS) delivered to the ventral capsule/ventral striatum (VC/VS) target for obsessive-compulsive disorder (OCD) decreased beta-gamma cross-frequency coupling (CFC) in electroencephalographic (EEG) recordings from the occipital cortex. They suggested that this may represent changes in bottom-up visual processing that may make disorder-related stimuli exceptionally salient. They further noted a recent report of DBS-induced changes in motor cortex beta-gamma coupling during DBS for Parkinson's disease (PD) (2) as potentially supporting that hypothesis.

OCD, however, is different from PD in several respects. First, although there is a basalganglia-linked hypothesis of OCD (3), there is no clear degenerative lesion as there is in PD. Second, multiple groups have identified abnormal cortical and subcortical beta rhythms in PD (4), whereas there is no robust or well-replicated EEG signature of OCD in any frequency band. Finally, psychiatric diagnoses have profound internal heterogeneity, and this may be specifically worse in DBS cases given how rare and treatment-resistant these patients are (5,6). The observed effect of Bahramisharif et al. (1) may be driven by specific DBS settings or by unmodeled patient characteristics, including patients' level of occipital CFC before surgery. Alternately, it may be specific to OCD or a clinical subphenotype that is more frequently selected for DBS at the authors' center. Those alternatives are difficult to assess because few centers are able to accumulate sufficient DBS patients to conduct replication studies.

We have a similar ongoing EEG study in VC/VS DBS, offering an opportunity to replicate the Bahramisharif et al. (1) analysis. Similar to the original authors, we recorded 60-channel, eyes-open, resting-state EEG from five patients who had previously received VC/VS DBS at

\footnotetext{
Address correspondence to Alik S. Widge, M.D., Ph.D., Massachusetts General Hospital and Harvard Medical School, Department of Psychiatry, 149 13th St, Room 2625, Charlestown, MA 02129; awidge@ partners.org.

See also associated correspondence: http://dx.doi.org/10.1016/j.biopsych.2015.12.014.

All other authors report no biomedical financial interests or potential conflicts of interest.
} 
our center and had a clinical response. The study protocol was approved by the Partners Healthcare Institutional Review Board, and all subjects provided informed consent to recordings. Our sample differed in that four of the five patients were implanted for major depressive disorder rather than OCD, and one of the five patients had DBS programmed at $40 \mathrm{~Hz}$ to $50 \mathrm{~Hz}$. The duration of DBS discontinuation was briefer; Bahramisharif et al. (1) studied patients who withdrew from DBS for a full week, whereas for safety reasons, our sample spent only 2 hours with DBS off before EEG collection. We replicated precisely the original authors' filtering, referencing, and analysis, using the FieldTrip toolbox for preprocessing (7) and MATLAB (The MathWorks, Inc., Natick, Massachusetts) code graciously provided by Dr. Bahramisharif for CFC quantification at the occipital midline $(\mathrm{Oz})$ electrode.

In this independent sample, we were unable to replicate the results of Bahramisharif et al. (1) (Figure 1). Within the zone of significant change identified by the original article (white box in Figure 1), VC/VS slightly increased CFC, opposite to the authors' original findings. This increase was not statistically significant $(p=.209$, two-sample Kolmogorov-Smirnov test). It was, however, strongest at $\mathrm{Oz}$, with smaller mean differences and higher $p$ values at other midline electrodes $(\mathrm{Cz}, \mathrm{Fz}, \mathrm{Pz})$. We further tested for specific coupling between alpha $(8-12 \mathrm{~Hz})$, beta $(13-30 \mathrm{~Hz})$, and theta $(5-8 \mathrm{~Hz})$ bands and found no significant effects even before multiple-comparisons correction (all $p>.1$ ). Overall, our results are most consistent with a lack of VC/VS DBS effect on CFC of midline electrodes.

This conflicting result could be explained by diagnoses, since most of our sample patients did not have OCD. The single OCD subject in our analysis, however, showed a stronger on > off CFC pattern than the group as a whole. It might also be that the results of Bahramisharif et al. (1) are specific to high-frequency DBS. Excluding the subject with $40 \mathrm{~Hz}$ to $50 \mathrm{~Hz}$ DBS, however, drove the difference between DBS-on and DBS-off closer to 0 (to .003 normalized units) in our cohort. Next, since our study involved only a brief DBS discontinuation, the original authors' findings might only be visible after their week-long withdrawal. While we cannot rule this out, animal studies of DBS-like stimulation suggest that immediately after stimulation withdrawal, brain electric potentials rebound in a direction opposite to the DBS effect (8). Our short time window should have revealed an even stronger CFC effect than Bahramisharif et al. (1) observed.

We suggest that these opposing CFC findings more likely depend on unmodeled characteristics of the two samples. This could include preoperative clinical or electrophysiologic phenotypes, slight surgical variation in electrode placement, choice of active DBS contacts, or even subtle features of the testing environment. To the latter point, individual peak frequencies and phase locking of occipital rhythms have been shown to change quickly in response to attentional processing and expectancy (9). Subjects' internal state, such as the negative ruminations of major depressive disorder or obsessions of OCD, may have driven the findings in either sample. More importantly, while DBS likely does have strong cortical effects, we believe our data suggest that the specific finding of altered CFC cannot be considered a general mechanism of action. Further study, including presurgical baseline observations, will be needed to identify the origins and clinical significance of DBS-induced CFC changes. Multicenter coordination on study protocols 
would help to mitigate the small sample size issues inherent to the Bahramisharif et al. (1) study and our replication.

\section{Acknowledgments}

Acknowledgments and Disclosures

ASW, SZ, TD, ENE, and DDD acknowledge the support of the US Army Research Office and Defense Advanced Research Projects Agency under Cooperative Agreement Number W911NF-14-2-0045. ASW and EKM further gratefully acknowledge the support of the Picower Foundation and EKM of the National Institutes of Health under Grant No. 5R37MH087027. The views and conclusions contained in this document are those of the authors and should not be interpreted as representing the official policies, either expressed or implied, of the funding sources.

DDD and ENE have received research support from and served on advisory panels for Medtronic and Cyberonics. EKM serves on the scientific advisory board of Thync.

\section{References}

1. Bahramisharif A, Mazaheri A, Levar N, Schuurman PR, Figee M, Denys D. Deep brain stimulation diminishes cross-frequency coupling in obsessive-compulsive disorder. Biol Psychiatry. 2016; 80:e57-e58. [PubMed: 26166231]

2. de Hemptinne C, Swann NC, Ostrem JL, Ryapolova-Webb ES, San Luciano M, Galifianakis NB, Starr PA. Therapeutic deep brain stimulation reduces cortical phase-amplitude coupling in Parkinson's disease. Nat Neurosci. 2015; 18:779-786. [PubMed: 25867121]

3. Milad MR, Rauch SL. Obsessive-compulsive disorder: Beyond segregated cortico-striatal pathways. Trends Cogn Sci. 2012; 16:43-51. [PubMed: 22138231]

4. Little $S$, Brown P. What brain signals are suitable for feedback control of deep brain stimulation in Parkinson's disease? Ann N Y Acad Sci. 2012; 1265:9-24. [PubMed: 22830645]

5. Garnaat SL, Greenberg BD, Sibrava NJ, Goodman WK, Mancebo MC, Eisen JL, Rasmussen SA. Who qualifies for deep brain stimulation for OCD? Data from a naturalistic clinical sample. J Neuropsychiatry Clin Neurosci. 2014; 26:81-86. [PubMed: 24515679]

6. Widge AS, Deckersbach T, Eskandar EN, Dougherty DD. Deep brain stimulation for treatmentresistant psychiatric illnesses: What has gone wrong and what should we do next? Biol Psychiatry. 2016; 79:e9-e10. [PubMed: 26212895]

7. Oostenveld R, Fries P, Maris E, Schoffelen J-M. FieldTrip: Open source software for advanced analysis of MEG, EEG, and invasive electrophysiological data. Comput Intell Neurosci. 2010; 2011:156869. [PubMed: 21253357]

8. Ewing SG, Grace AA. Long-term high frequency deep brain stimulation of the nucleus accumbens drives time-dependent changes in functional connectivity in the rodent limbic system. Brain Stimul. 2013; 6:274-285. [PubMed: 22981894]

9. Samaha J, Bauer P, Cimaroli S, Postle BR. Top-down control of the phase of alpha-band oscillations as a mechanism for temporal prediction. Proc Natl Acad Sci U S A. 2015; 112:8439-8444.

[PubMed: 26100913] 

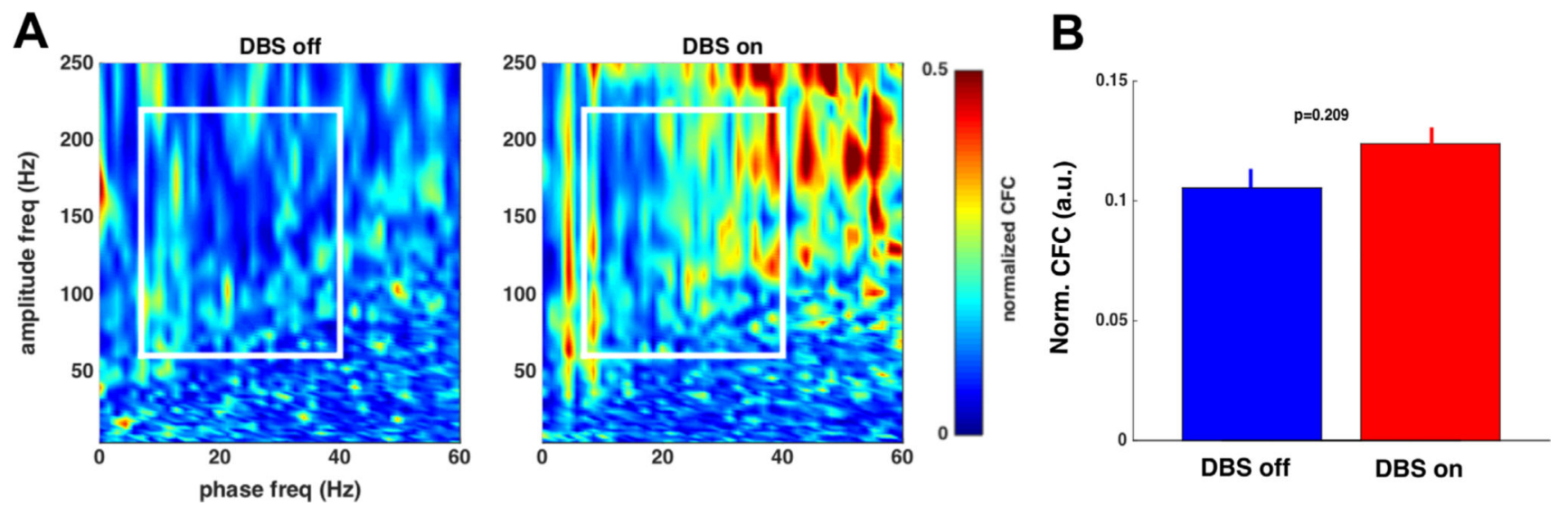

Figure 1.

(A) Cross-frequency coupling (CFC), averaged across subjects, normalized to maximum of the analysis window (white box). Preprocessing and analysis parameters are identical to Bahramisharif et al. (1). We observed a moderate but nonsignificant increase in CFC with deep brain stimulation (DBS) on, opposite to the original authors' effect. (B) Average CFC values over the window highlighted in (A), with error bars representing the standard error of the mean over all analyzed patients $(n=5)$. The two conditions are not significantly different ( $p=.209$ ) by a two-sample Kolmogorov-Smirnov test. a.u., arbitrary unit; freq, frequency; Norm., normalized. 